\title{
avß6-Fyn Kinase Promotes Epithelial Phenotype in SYF Cells
}

\author{
CASEY LEE and DANIEL M. RAMOS \\ Department of Orofacial Sciences, University of California at San Francisco, San Francisco, CA, U.S.A.
}

\begin{abstract}
The 5-year survival rate for patients with oral cancer remains at 50\%, in large part due the high rate of posttreatment recurrence. In this study, we transfected epithelialspecific integrin $\alpha v \beta 6$ and Fyn-kinase, a member of the Srcfamily kinases, into embryonic murine fibroblasts. In oral cancer, expression of $\alpha v \beta 6$ is neo-expressed. Using a variety of in vitro assays, including cell migration and multicellular spheroid formation, we determined that these embryonic fibroblasts expressing $\alpha v \beta 6$ and Fyn-kinase were able to acquire an epithelial phenotype. This is in direct contrast to human oral SCC, where expression of $\alpha v \beta 6$ with Fyn-kinase promotes epithelial to mesenchymal transition. This demonstrates that signaling pathways can be species-specific.
\end{abstract}

Oral cancer is the sixth most common cancer worldwide. In India, oral cancer accounts for more than $50 \%$ of all malignancies. However, in the United States, oral cancer accounts for roughly $5 \%$ of new cancer cases and $96 \%$ of these are squamous cell carcinoma (SCC) (1). This year, 49,670 new cases of oral cancer will be diagnosed in the United States, and more than 9,700 patients will die from this disease (2).

Oral SCC consists of a morphologically-heterogeneous population of cells. This heterogeneity likely contributes to the varied response to traditional treatment (ionizing radiation and chemotherapy). Our laboratory previously showed that neoexpression of the integrin $\alpha v \beta 6$ is one of the more notable changes that occur in human oral epithelial transformation (3).

In this study, when epithelial integrin $\alpha v \beta 6$ and an activated Fyn were expressed in murine embryonic fibroblasts they emulated the morphology and motility found in epithelial cells, suggesting mesenchymal to epithelial cell transition MET) had occurred.

Correspondence to: Daniel M. Ramos, DDS, Ph.D., Department of Orofacial Sciences, University of California, 513 Parnassus Ave, S612, Box 0422, San Francisco, CA 94143-0422, U.S.A. Tel: +1 4155024905, Fax: +1 8007836653, e-mail: Daniel.ramos@ucsf.edu

Key Words: SYF cells, multicellular spheroid formation, MAPK, Fyn kinase, migration.

\section{Materials and Methods}

Cell lines. Cells deficient for Src, Yes, and Fyn (SYF) were generated in 1997 from mouse embryos with null mutations in both alleles of genes for the Src family protein tyrosine kinases, Src, Yes, and Fyn (4). The cells were immortalized using a retroviral vector transducing the SV40 large T-antigen and carry neomycin resistance (4).

Transfection of the $\beta 6$ integrin into SYF cells established the SYF $\beta 6$ cell line. Similarly, the transfection of the constitutively active Fyn into SYF cells resulted in formation of the SYF $36 C A F Y N$ cell line (5). The full-length $\beta 6$ cDNA was a gift from Dr. Dean Sheppard (University of California at San Francisco). The cDNA for the constitutively active Fyn (CA-Fyn) was a gift from Drs. H. Kawakatsu (University of California at San Francisco) and Dean Sheppard. The cDNA was stably expressed in the murine SYF cell line, using the Retro-X system (Takara Bio, Mountain View, CA, USA) (5). Cells were grown in Dulbecco's modified Eagle's medium (DMEM) with $10 \%$ fetal bovine serum (5).

Antibodies. Rabbit monoclonal antibodies to phospho-p44/43 mitogen-activated protein kinase (MAPK), extracellular signalregulated kinase (ERK) (Thr202/Tyr204) (antibody \#9101), MAPK (p44/42 MAPK (ERK1/2) \#9102), $\beta$-actin (clone13E5), and horseradish peroxidase (HRP)-conjugated anti-rabbit (clone7074) and HRP-anti-mouse (CST \#7076) antibodies were purchased from Cell Signaling Technology Inc. (Danvers, MA, USA).

Migration assay. Cell migration was evaluated using a $6.5 \mathrm{~mm}, 8$ $\mu \mathrm{M}$ pore-size Transwell ${ }^{\circledR}$ filter migration system (\#3422; Corning Life Sciences, Durham, NC, USA). The undersurface of the filter was coated with $10 \mu \mathrm{g} / \mathrm{ml}$ of fibronectin overnight at $37^{\circ} \mathrm{C}$. The lower chamber contained $500 \mu \mathrm{l}$ of fibroblast-conditioned medium as a chemoattractant. The SYF cells (SYF, SYF 36 , and SYF 36 CAFYN; $2 \times 10^{5}$ ) were plated onto the upper surface in serum-free medium. The assay was carried out without serum at $37^{\circ} \mathrm{C}$ for $12 \mathrm{~h}$ in the presence of $5 \% \mathrm{CO}_{2}$. The filters were then fixed using $2 \%$ paraformaldehyde in phosphate-buffered saline (PBS), and the upper surface wiped clean using cotton swabs. The filter was immersed in crystal violet for 20 minutes (1\% paraformaldehyde, $0.5 \%$ crystal violet, and $20 \%$ methanol in PBS). Filters were rinsed twice with PBS, air-dried, evaluated visually and the number of cells crossing the membrane were determined by counting five random fields/filter (in triplicate).

Generation of multicellular spheroids (MCS). Tissue culture plates (Santa Cruz Biotechnology, Dallas, TX, USA) were coated with a thin layer of $0.6 \%$ agarose solution and air-dried overnight at room 
temperature. Cells were harvested from monolayer cultures using $0.25 \%$ trypsin-EDTA and rinsed twice to remove serum. The cells were then placed on agarose-coated plates for $48 \mathrm{~h}$ at $37^{\circ} \mathrm{C}$ in $5 \%$ $\mathrm{CO}_{2}$ in serum-free media. The size of the spheroids was traced, analyzed and quantified using NIH Image J (6). Data are expressed as relative value units (rvu).

Western blot. The cells were grown as a monolayer or MCS in serumfree media for $24 \mathrm{~h}$. The cells were then lysed and total protein was extracted using RIPA lysis buffer [50 mM Tris pH $8,150 \mathrm{mM} \mathrm{NaCl}$, $0.2 \%$ sodium dodecyl sulfate (SDS), $1 \%$ deoxycholic acid, $1 \%$ Triton $\mathrm{X}$-100. complete protease inhibitor tablet (Sigma-Aldrich, Carlsbad, CA, USA). Proteins were resolved using SDS-polyacrylamide gel electrophoresis (PAGE) $(7.5 \%)$ and transferred to a polyvinylidene fluoride (PVDF) membrane using a semi-dry apparatus (Bio-Rad, Hercules, CA, USA). PVDF membranes were subsequently incubated with monoclonal antibodies listed above overnight at $4^{\circ} \mathrm{C}$. After washing, the blots were incubated with HRP-conjugated anti-rabbit antibodies for $1 \mathrm{~h}$. The blots were treated with enhanced chemiluminescence substrate (Thermo Scientific Pierce; Rockford, IL, USA) and exposed to X-ray film (Santa Cruz Biotechnology, Dallas, TX, USA). Western blot images were quantified using NIH Image $J(6)$. $\beta$-Actin or tubulin were used as controls to quantify the bands in each image. The images were digitally captured and assigned rvu based on the intensity of the signal. Image $J$ toolbar was used to draw a rectangle around the control lane (tubulin or actin) and sample lanes. Relative area sizes were evaluated from the generated peaks. Each area was expressed as a percentage of the total size of all of the highlighted peaks. Each subsequent percentage was then divided by the percentage of the assigned control to generate expression as rvu.

\section{Results}

$\beta 6$ Integrin and active Fyn promote epithelial cell morphology in undifferentiated murine fibroblasts. The murine SYF, SYF $\beta 6$ and SYF $\beta 6 C A F Y N$ cells were grown on tissue culture plastic for $120 \mathrm{~h}$ under low serum conditions $(1 \%)$. Both the SYF and the SYF $\beta 6$ cells retained their fibroblastic appearance over the course of the assay and never reached confluence (Figure 1). However, the SYF 36 CAFYN cells contracted and took on a compact/epithelial-like morphology and did reach confluence (Figure 1). In the absence of integrin $\alpha v \beta 6$ and its ligand Fyn kinase, the murine cells retained their mesenchymal-like phenotype.

Differential formation of MCS by murine SYF cells. We used an MCS assay to evaluate the ability of the murine SYF cells to form three-dimensional structures in vitro. The SYF, SYF $\beta 6$ and the SYF $\beta 6 C A F Y N$ cells were grown, serumfree, on agarose-coated tissue-culture plates (7). The cultures were monitored every $12 \mathrm{~h}$ and at $48 \mathrm{~h}$ the experiment was terminated. SYF cells did not readily form cell-cell clusters, but rather formed the smallest MCS (Figure 2). $\beta 6$ Integrin co-expressed with Fyn kinase resulted in formation of the largest MCS. MCS formation was as follows: SFY $\beta 6 C A F Y N \gg$ SYF $\beta 6>>$ SYF cells (Figure 2).
Cell migration as a response to $\alpha v \beta 6$ integrin. In order to examine cell migration, the under surface of Transwell filters was coated with fibronectin. Cell migration occurred at different rates across Transwell filters coated with $10 \mu \mathrm{g} / \mathrm{ml}$ of fibronectin, with the SYF cells migrating the least (Figure 3 ). The forced expression of $\beta 6$ integrin increased migration by $40 \%$ (Figure 3 ). When both $\beta 6$ integrin plus a constitutively active Fyn kinase were expressed, cell migration doubled (Figure 3). These results indicate that expression of $\beta 6$ integrin with Fyn kinase can promote cell migration in embryonic murine fibroblasts.

Differential expression of pMAPK in SYF MCS. MAPKs are often thought of as biological master switches involved in controlling a wide array of cellular responses including proliferation, gene expression, differentiation, cell survival, and apoptosis (8).

The SYF, SYF $\beta 6$ and SYF $\beta 6 C A F Y N$ cells were grown on tissue culture plates or on agarose-coated plates for $48 \mathrm{~h}$ to evaluate MAPK phosphorylation by western blotting.

The total expression of MAPK was approximately equivalent in all cell lines whether grown as a monolayer or as MCS (Figure 4). However, phosphorylation of MAPK was clearly different between the different cell lines (Figure 4). Surprisingly, when grown as MCS, pMAPK was greatest in SYF cells (Figure 4). Phosphorylation of MAPK was barely detectable in SYF $\beta 6$ and SYF $\beta 6$ cells (Figure 4). These results suggest that activation of MAPK in murine embryonic fibroblasts occurs independently of $\beta 6$ and FYN.

\section{Discussion}

Our previous work focused on oral cancer cell behavior, specifically on the role of the $\alpha v \beta 6$ and its signaling potential $(5,9,10)$. In this study, we identified factors that promote the epithelial phenotype in Src-kinase-null murine embryonic fibroblasts. Metaplasia in this murine system requires both $\beta 6$ integrin and Fyn kinase. Our results demonstrate that the integrin $\beta 6$ and its Fyn kinase promote mesenchymal to epithelial transition (MET) in embryonic murine fibroblasts.

Integrins are heterodimeric glycoproteins consisting of an alpha- and a beta-chain (11). Each subunit contains a long extracellular domain, a transmembrane region and cytoplasmic domain capable of connecting to the actin cytoskeleton and triggering signal transduction events into the cell (12).

Our group previously demonstrated that cell migration was a direct consequence of $\beta 6$ integrin expression in oral SCC cells $(5,10)$. We previously showed that the engagement of $\beta 6$ integrin stimulated secretion of matrix metalloproteinases 2, 3 and 9, and localization and stabilization capable of localized degradation of the extracellular matrix (3). Our 


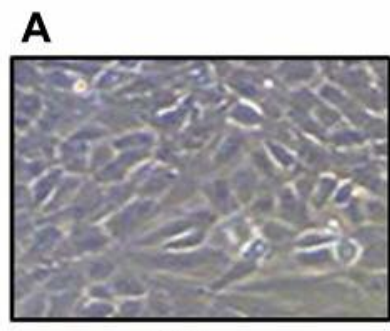

SYF
B

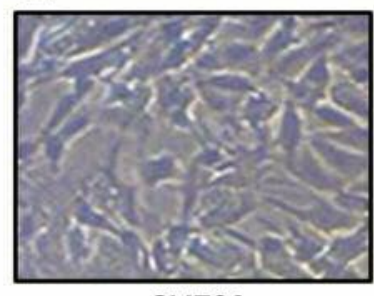

SYF $\beta 6$

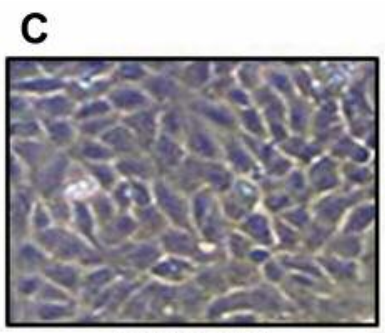

SYFß6CAFyn

Figure 1. Expression of $\beta 6$ and Fyn kinase promotes mesenchymal to epithelial transition in SYF cells (with null mutations in both alleles of genes for the Src family protein tyrosine kinases, Src, Yes, and Fyn). SYF cells (A), SYF cells transfected with $\beta 6$ integrin (SYF $\beta 6)(B)$ and SYF cells with constitutively active Fyn (SYFB6CAFyn) (C) were grown on serum-free tissue culture plates for 48 h. SYF and SYF $\beta 6$ cell lines retained a-fibroblast shape, while SYFß6CAFyn cells contracted, becoming more epithelial in morphology. Magnification used: 200x.

A

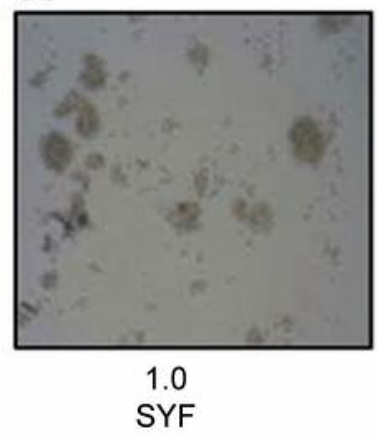

B

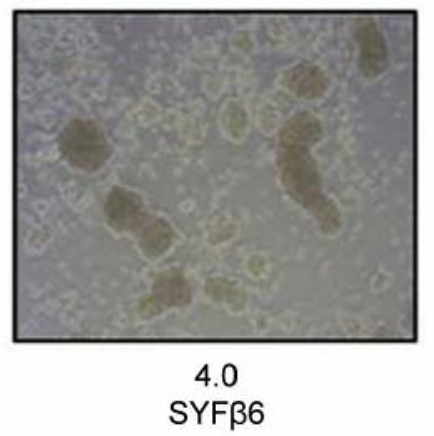

C

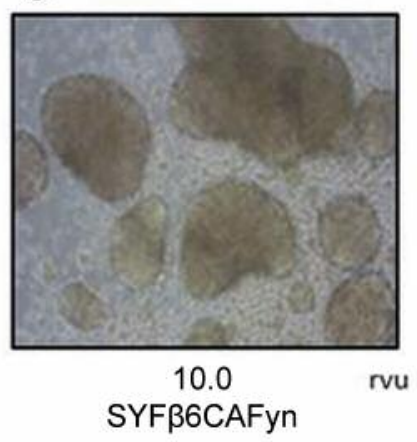

Figure 2. Multicellular spheroid (MCS) formation is regulated by $\beta 6$ and Fyn kinase. SYF cells (with null mutations in both alleles of genes for the Src family protein tyrosine kinases, Src, Yes, and Fyn) (A), SYF cells transfected with $\beta 6$ integrin (SYF $\beta 6)$ (B) and SYF cells with constitutively active Fyn (SYFß6CAFyn) (C) were grown on agarose-coated plates for 120 h. rvu: Relative value units. Magnification used: $200 \times$.

previous work also showed that the $\beta 6$ integrin does not mediate initial cell attachment but rather plays a substantial role in secondary adhesion events such as cell migration (3).

The MAPK/ERK pathway takes extracellular information and communicates this information to the nucleus of the cell. p42 and p44 MAPKs are activated through integrin clustering (13). In this murine SYF system, it appears that pMAPK suppresses MET whether the cells are grown as a monolayer or MCS. In human oral cancer, expression of $\beta 6$ is associated with tumor aggressiveness and invasion. In the murine system, we found the exact opposite occurs (14).

In the current study, pMAPK was expressed the greatest by the SYF cells. In this system, the expression of $\beta 6$ with CAFYN suppressed MAPK phosphorylation, which is one of the master switches in cell signaling. This indicates that not all major signaling processes occur in all species.

In this study, murine embryonic fibroblasts were genetically modified to not express any member of the Src family (Src, Yes and Fyn). They retained their normal fibroblast-like morphology except in cells in which integrin $\beta 6$ and a

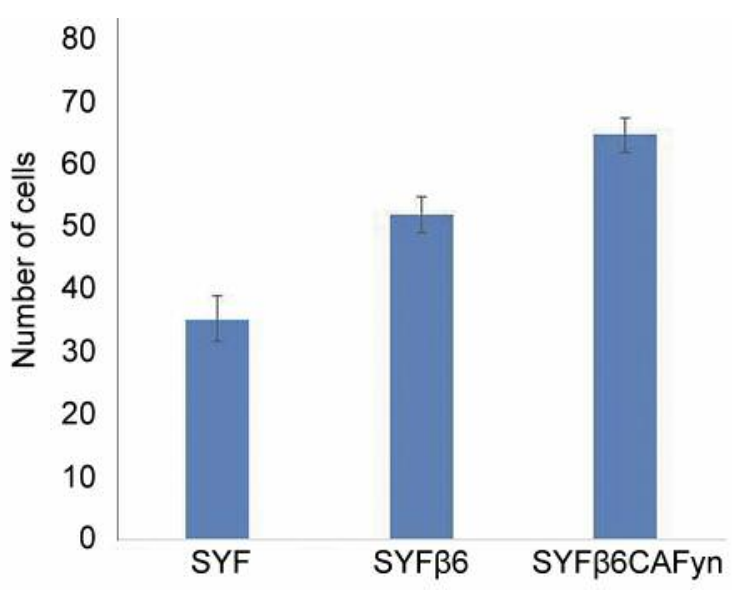

Figure 3. Invasion is modulated by $\beta 6$ and Fyn kinase. SYF cells (with null mutations in both alleles of genes for the Src family protein tyrosine kinases, Src, Yes, and Fyn), SYF cells transfected with $\beta 6$ integrin (SYF 36 ) and SYF cells with constitutively active Fyn (SYF $\beta 6 C A F y n)$ were plated onto Transwell filters in which the bottom surface was coated with $3 \mu \mathrm{g} / \mathrm{ml}$ of fibronectin. The cells were allowed to migrate onto fibronectin substrate for $24 \mathrm{~h}$, after which the number of cells traversing the filter was determined. 
A

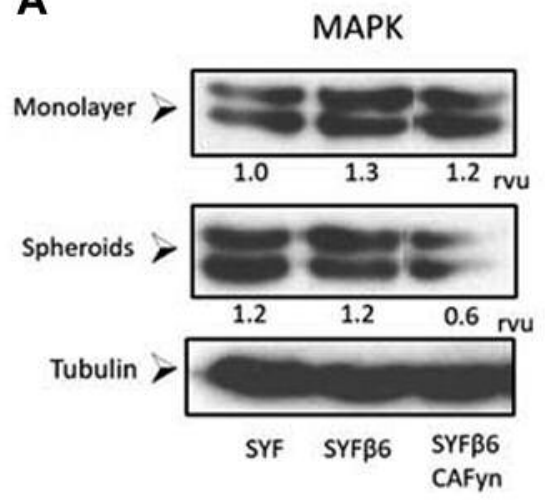

B

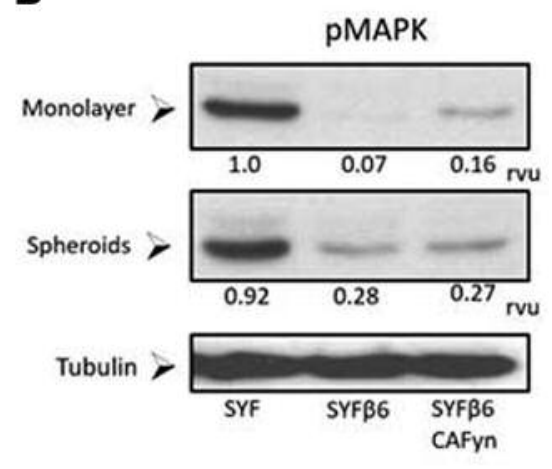

Figure 4. Activation of mitogen-activated protein kinase (MAPK) occurs independently of $\beta 6$ or activation of FYN. SYF cells (with null mutations in both alleles of genes for the Src family protein tyrosine kinases, Src, Yes, and Fyn), SYF cells transfected with $\beta 6$ integrin (SYF $\beta 6)$ and SYF cells with constitutively active Fyn (SYF $\beta 6 C A F y n$ ) were grown as monolayers or multicellular spheroid for 24 h and evaluated by western blotting for activation of MAPK (pMAPK). The blots were quantified and assigned relative value units (rvu) using NIH Image J.

constitutively active Fyn were co-expressed. This cotransfection resulted in the murine cells taking on an epithelial cell morphology, increasing cell migration and enhancing MCS formation (15). This indicates that cell morphology, migration, and MCS formation are directed by the interaction between $\beta 6$ and Fyn, but pMAPK is independent of this interaction. In the murine embryonic fibroblast system, the expression of $\alpha v \beta 6$ with Fyn-kinase promotes mesenchymal to epithelial transition. This is in direct contrast to human oral SCC, where expression of $\alpha v \beta 6$ with Fyn-kinase promotes epithelial to mesenchymal transition. This demonstrates that signaling pathways can be species-specific.

\section{References}

1 Oral Cancer Fact Sheet. NIH, 2010. Available from: https://report.nih.gov/NIHfactsheets/Pdfs/OralCancer(NIDCR).p df (last accessed 15-11-2017).

2 Cancer Stat Facts: Oral Cavity and Pharynx Cancer, 2014. Available from: https://seer.cancer.gov/statfacts/html/oralcav.html

3 Ramos DM, But M, Regezi J, Schmidt BL, Atakilit A, Dang D, Ellis D, Jordan R and Li X: Expression of integrin beta 6 enhances invasive behavior in oral squamous cell carcinoma. Matrix Biol 21(3):297-307, 2002.

4 Klinghoffer RA, Sachsenmaier C, Cooper JA and Soriano P: Src family kinases are required for integrin but not PGDFR signal transduction. EMBO J 18(9): 2459-2471, 1999.

5 Li X, Yang Y, Hu Y, Dang D, Regezi J, Schmidt BL, Atakilit A, Chen B, Ellis D and Ramos DM: $\alpha v \beta 6$-Fyn signaling promotes oral cancer progression. J Biol Chem 278(43): 41646-41653, 2003.

6 Rasband WS: ImageJ v1.6, U.S. National Institutes of Health, Bethesda, Maryland, USA, 1997-2015. http://imagej.nih.gov/ij/

7 Lee C, Lee C, Atakilit A, Siu A and Ramos DM: Differential spheroid formation by oral cancer cells. Anticancer Res 34(12): 6945-6949, 2014.
8 Hulit J, Suyama K, Chung S, Rinat K, Agiostratidou G, Shan W, Dong X, Williams T, Lisanti M, Knudsen K and Hazan R: NCadherin signaling potentiates mammary tumor metastasis via enhanced extracellular signal-regulated kinase activation. Cancer Res 67: 3106-3116, 2007.

9 Ramos DM, Chen BL, Boylen K, Stern M, Kramer RH, Sheppard D, Nishimura SL, Greenspan D, Zardi L and Pytela R: Stromal fibroblasts influence oral squamous-cell carcinoma cell interactions with tenascin-C: Int J Cancer 72(2): 369-376, 1997.

10 Ramos DM, Dang D and Sadler S: The role of the integrin $\alpha v \beta 6$ in regulating the epithelial to mesenchymal transition in oral cancer. Anticancer Res 29: 125-130, 2009.

11 Barczyk M, Carracedo S and Gullberg D: Integrins. Cell Tissue Res 339(1): 269-280, 2010.

12 Goodman SL and Picard M: Integrins as therapeutic targets. Trends Pharmacol. Sci 33(7): 405-412, 2012.

13 Ahmed N, Pansino F, Baker M, Rice G and Quinn M: Association between $\alpha v \beta 6$ integrin expression, elevated $\mathrm{p} 42 / 44$ $\mathrm{kDa}$ MAPK, and plasminogen-dependent matrix degradation in ovarian cancer. J Cell Biochem 84: 675-686, 2002.

14 Mogi S, Dang D, Van Waes C, Ellis D, Atakilit A and Ramos DM: The expression of integrin alpha(v)beta6 promotes the epithelial cell morphology and suppresses invasive behavior in transformed oral keratinocytes. Anticancer Res 25(2A): 751-755, 2005.

15 Lee C and Ramos DM: Regulation of multicellular spheroids by MAPK and FYN kinase. Anticancer Res 36(8): 3833-3838, 2016.
Received October 11, 2017

Revised November 13, 2017

Accepted November 16, 2017 